\title{
The Legal Nature of Smart Contracts
}

\author{
Salome Tezelashvili \\ PhD student of the faculty of law at the Caucasus International University, Department of Law and \\ Administration at the Shota Rustaveli National Science Foundation of Georgia
}

\section{ARTICLE INFO}

Article History:

Received 5.09.2021

Accepted 20.09.2021

Published 20.12.2021

Keywords:

Smart contract,

Kryptowaluty,

Computer algorithm

\section{ABSTRACT}

The article discusses about the smart contract, its concept and legal nature, as well as the place of smart contracts in the Technology Law, which means a discussision on the important issues covered by this topic. At the same time, smart contracts are compared to the usual standard contract, where their pros and cons are discussed. The importance and necessity of both types of contracts in relation to the current reality will also be discussed.

At the same time, the article discusses about the revolutions from where they begin and how long the world has passed before today's reality, why blockchain is considered as the fourth generation revolution and how important it is to develop and implement it.

The article also discusses about the types of contracts, which means how a standard contract can be divided, in the other words, we talk about consensual and real contracts. The definitions of each of them and their need related to the smart contracts are analysed in the article. Therefore, we use the relevant chapters and articles of civil law to be able to explain what is meant and to what extent it is possible to follow the same norms in the case of the smart contract. 


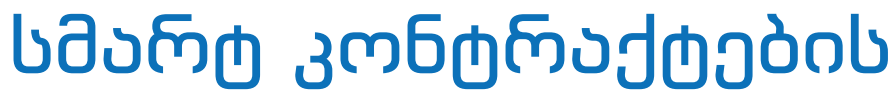

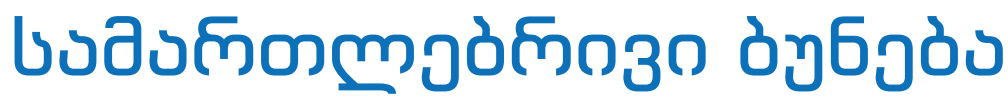

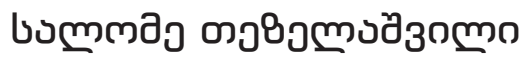

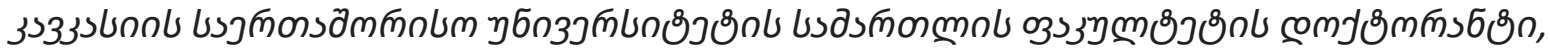

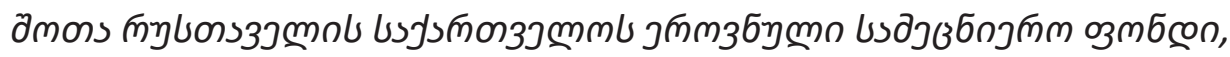

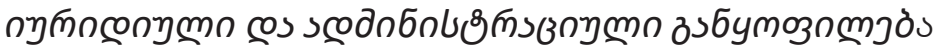

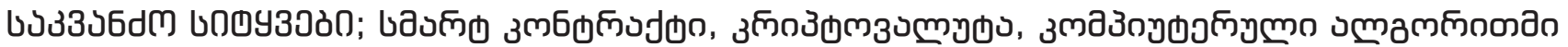

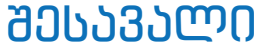

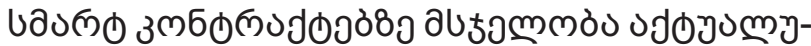

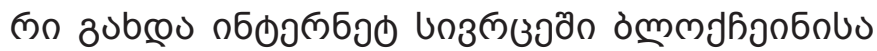

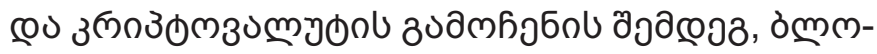

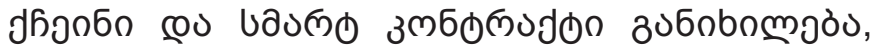

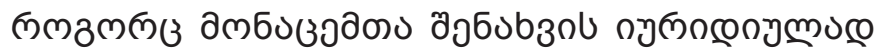

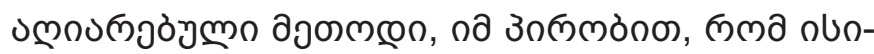

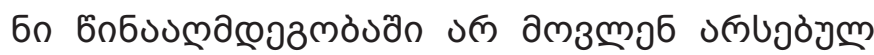

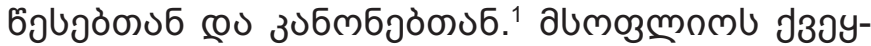

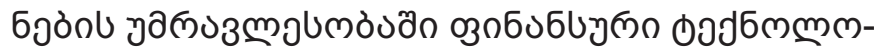

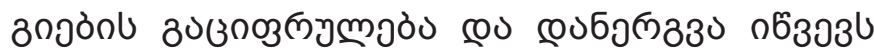

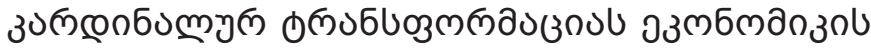

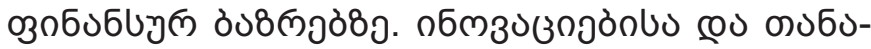

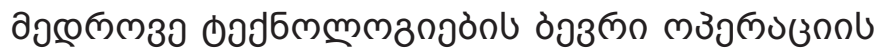

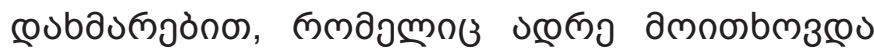

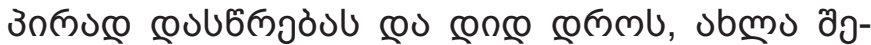

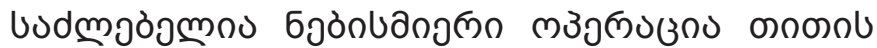

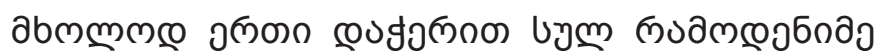

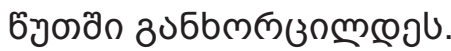

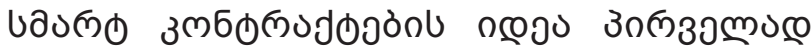

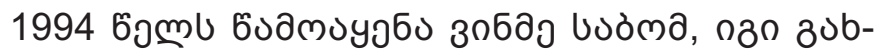

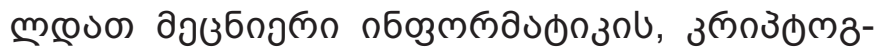

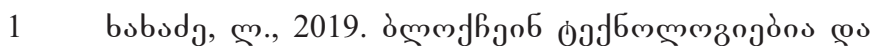

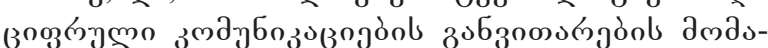

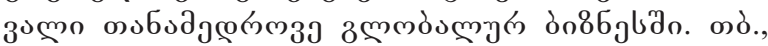
33. 485. https://dspace.tsu.ge/

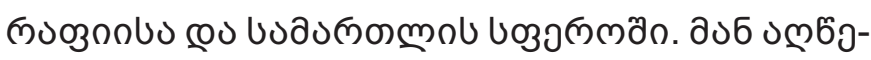

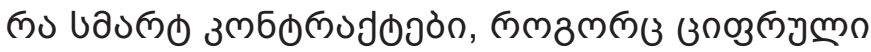

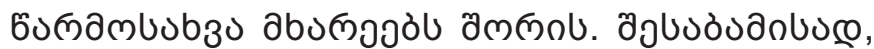

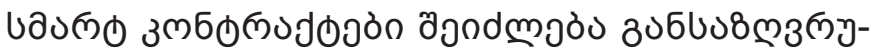

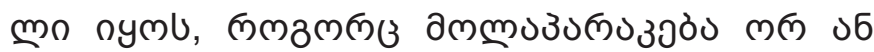

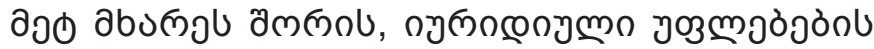

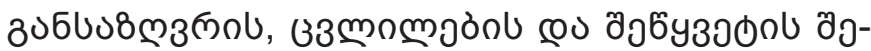

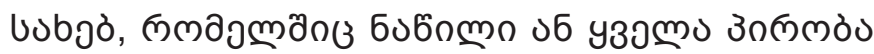

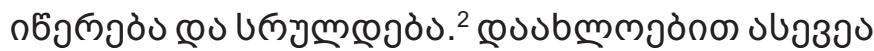

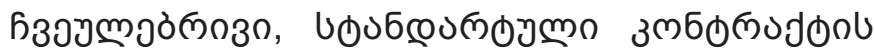

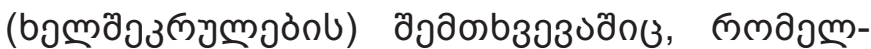

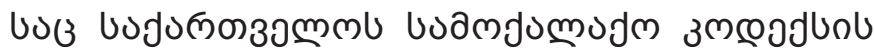

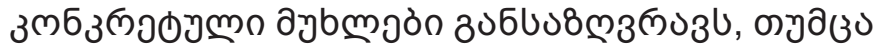

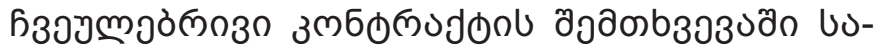

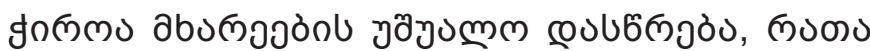

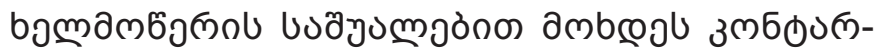

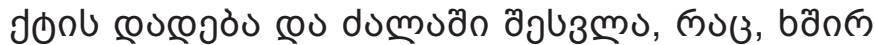

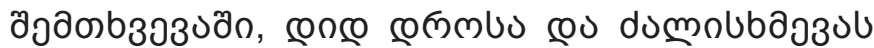

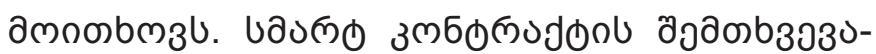

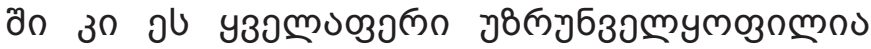

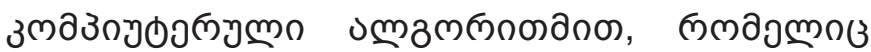

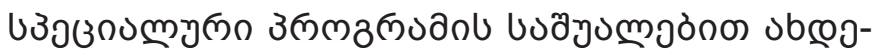

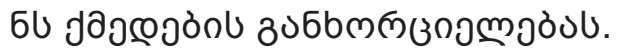

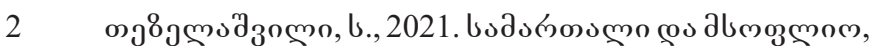

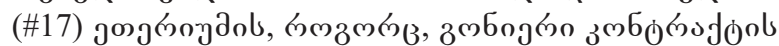

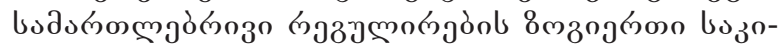
obo. os.., 33.168. 


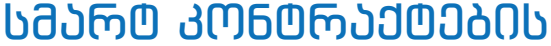

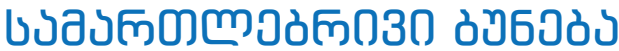

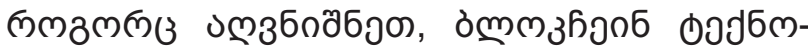

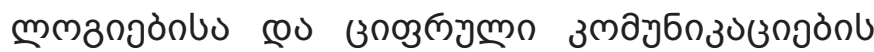

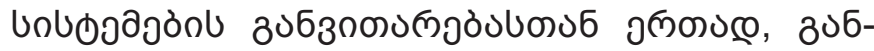

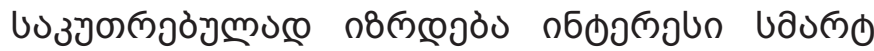

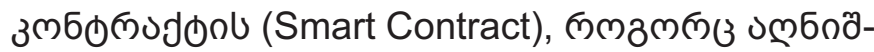

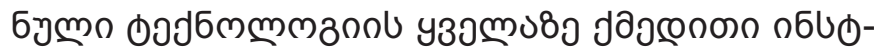

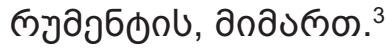

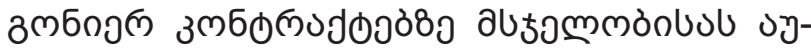

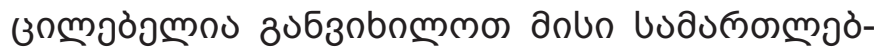

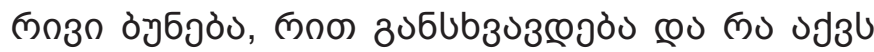

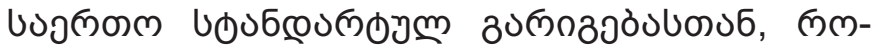

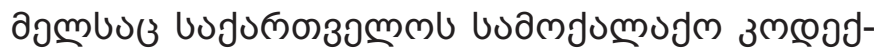

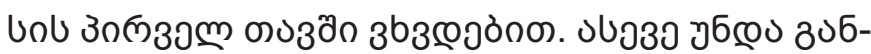

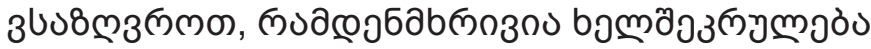

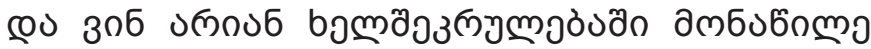
abง๓วอठ̀n.

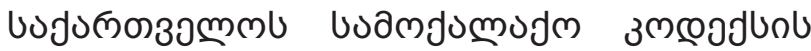

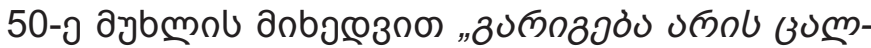

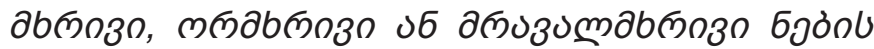

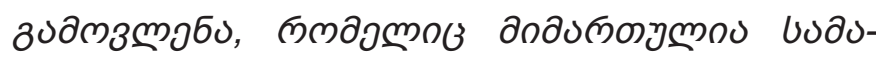

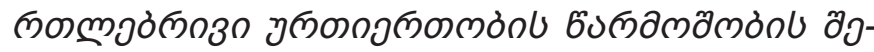

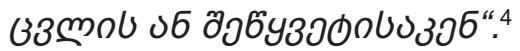

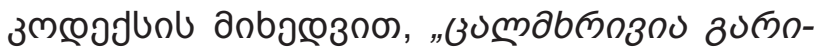

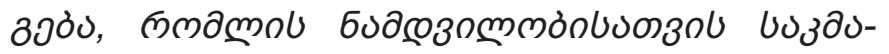

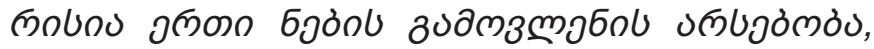

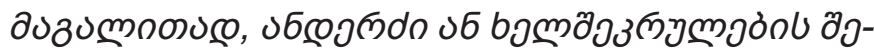

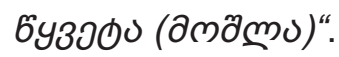

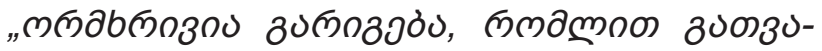

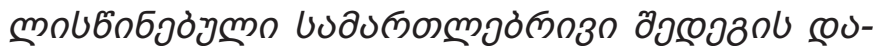

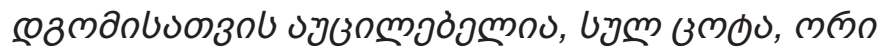

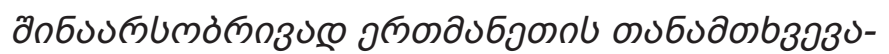

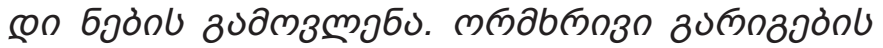

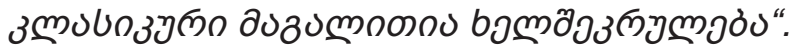

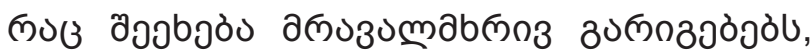

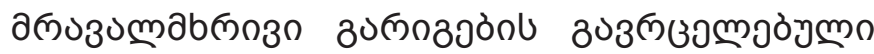

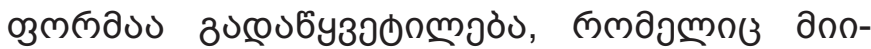

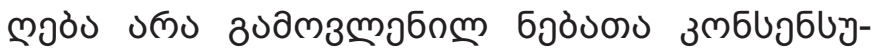

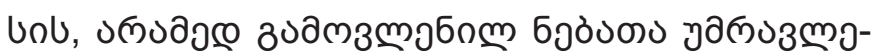

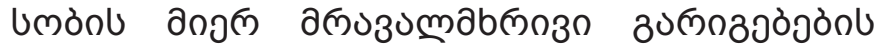

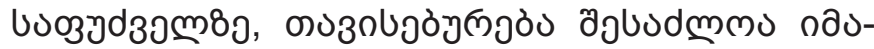

3

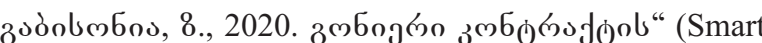

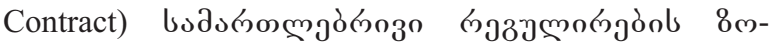

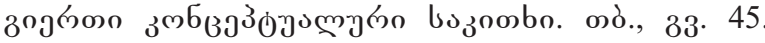
http://lawjournal.ge/wp

4

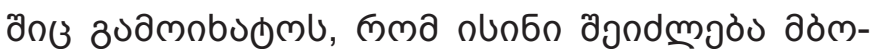

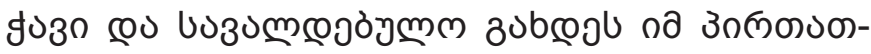

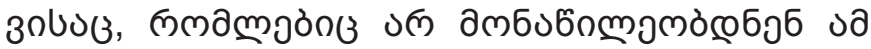

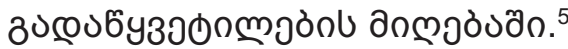

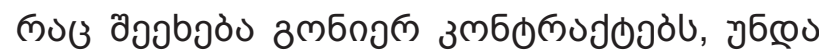

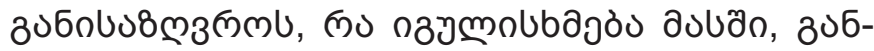

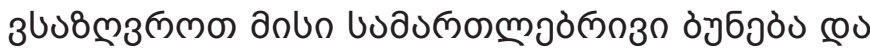

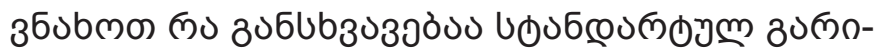

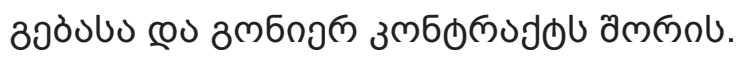

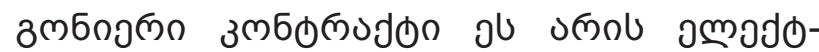

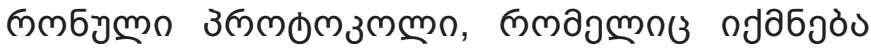

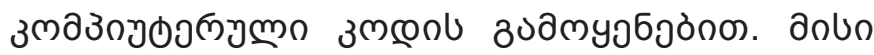

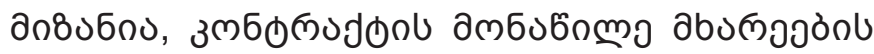

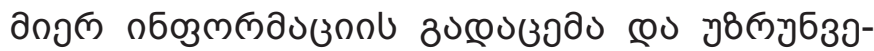

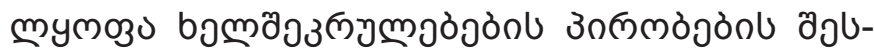

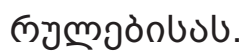

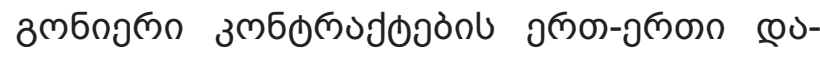

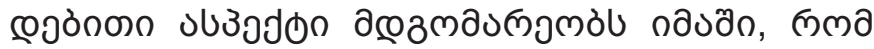

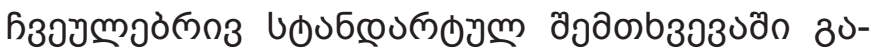

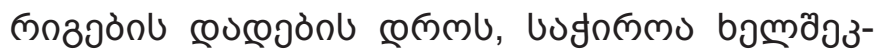

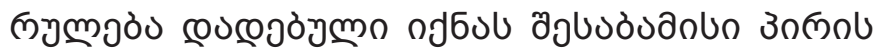

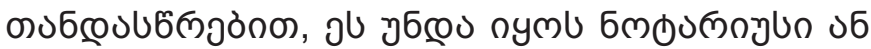

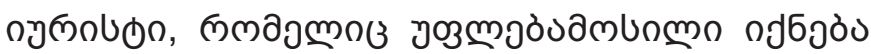

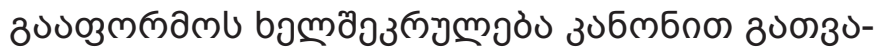

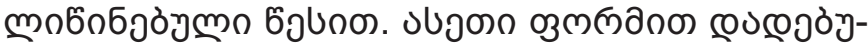

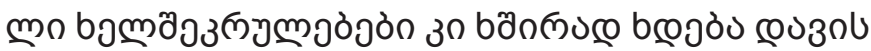

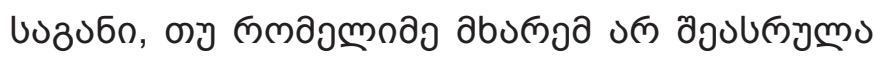

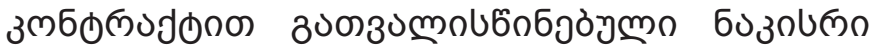

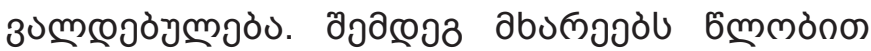

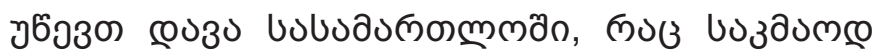

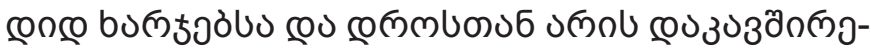

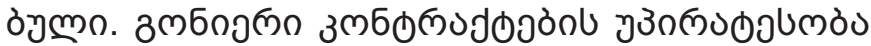

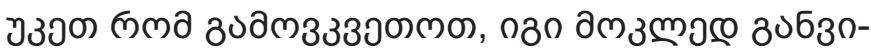

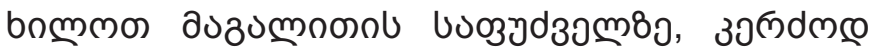

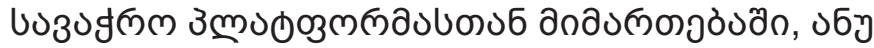

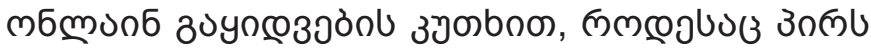

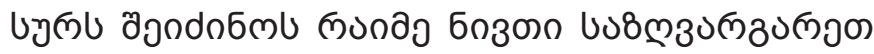

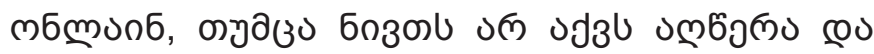

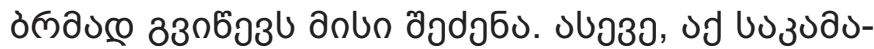

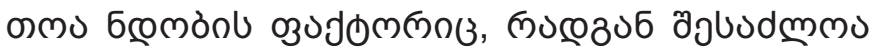

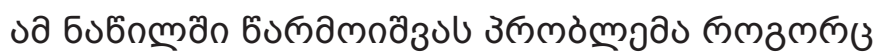

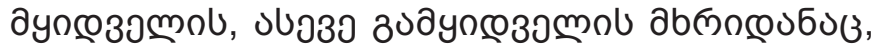

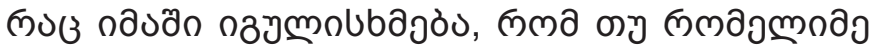

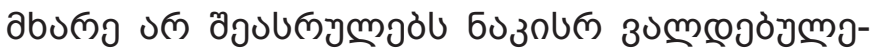

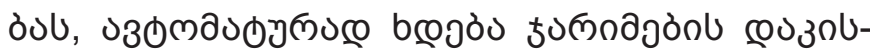

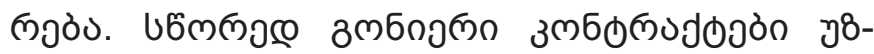

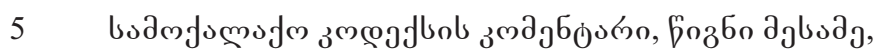

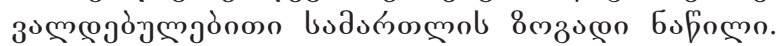
os., 2019. 33. 108, 109. http://lawlibrary.info/ge
} 


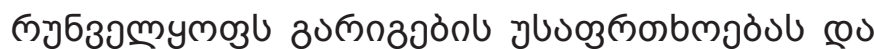

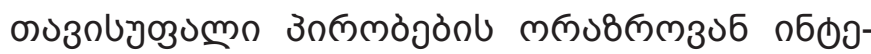

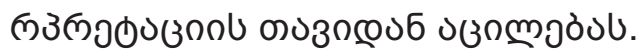

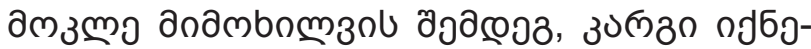

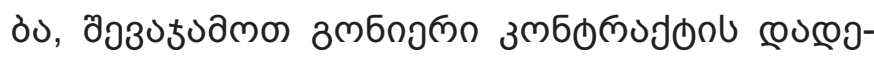

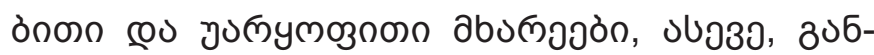

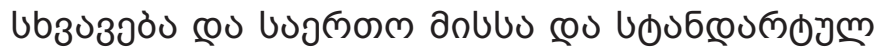

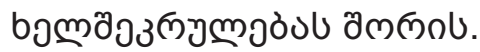

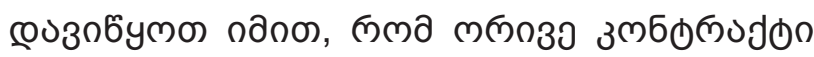

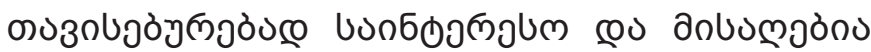

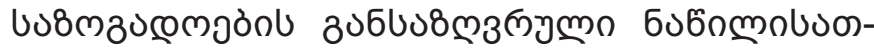

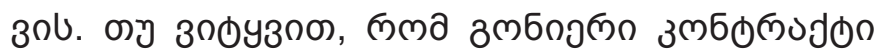

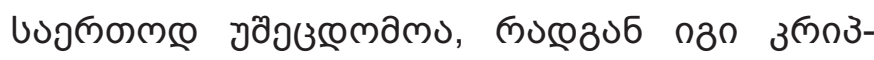

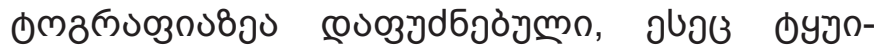

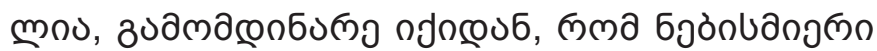

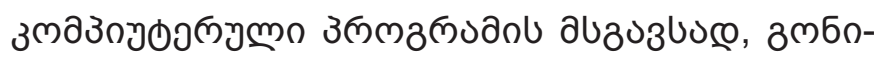

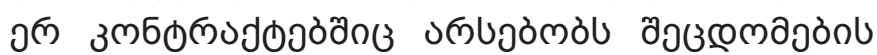

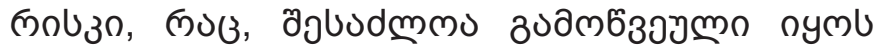

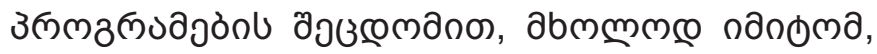

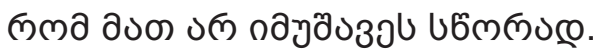

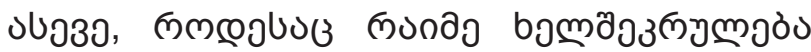

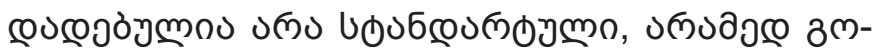

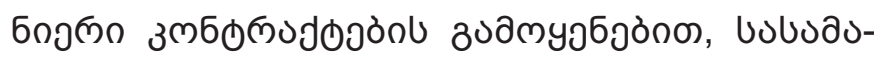

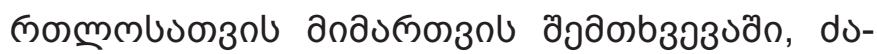

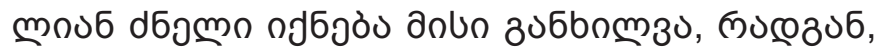

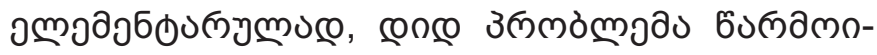

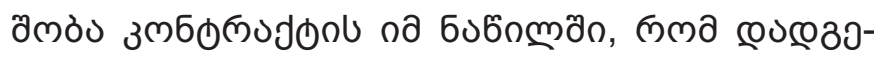

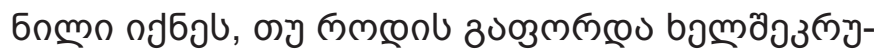

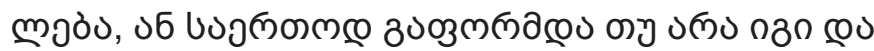

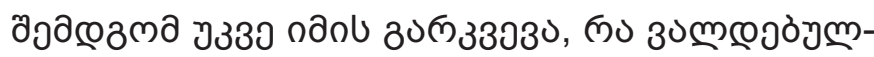

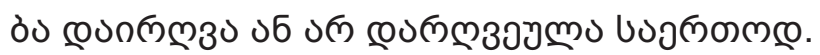

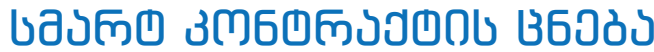

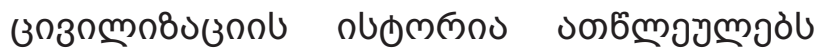

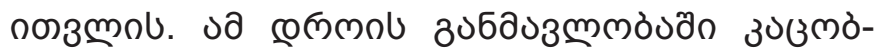

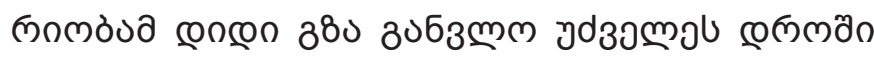

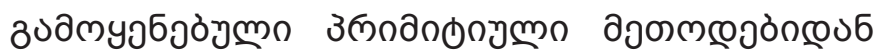

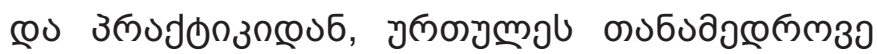

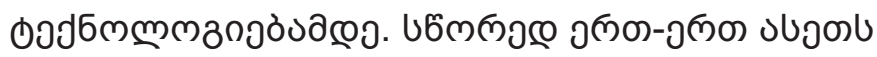

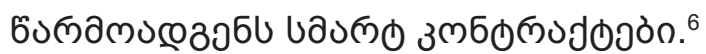

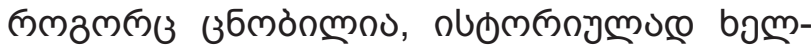

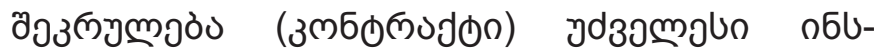

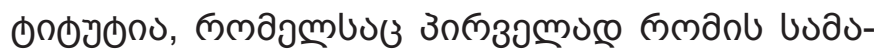

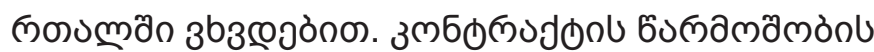

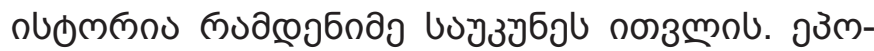

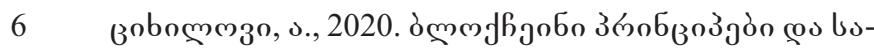

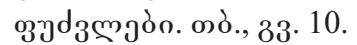

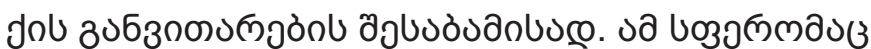

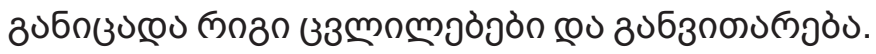

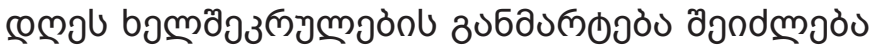

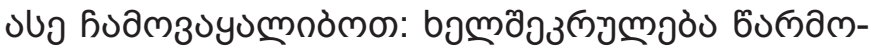

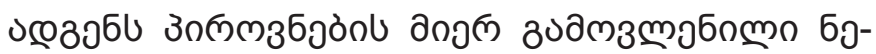

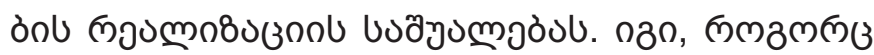

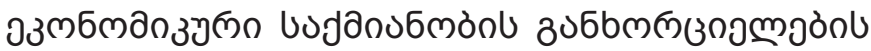

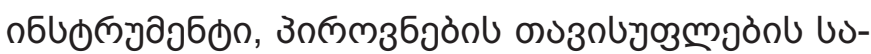

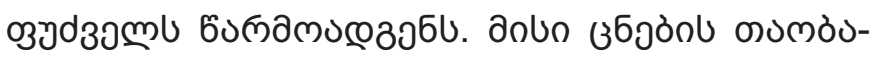

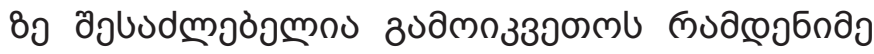

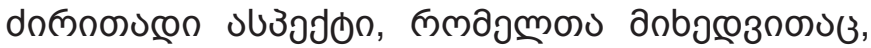

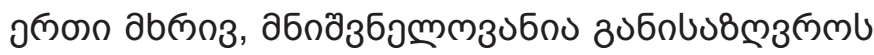

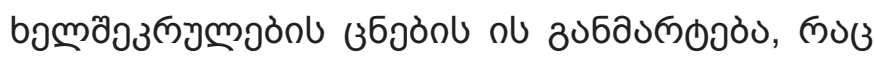

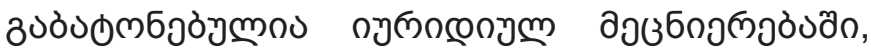

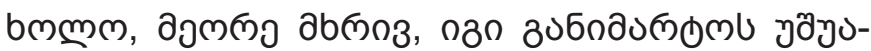

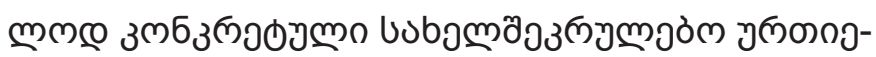

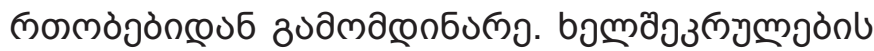

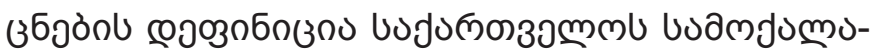

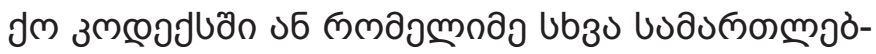

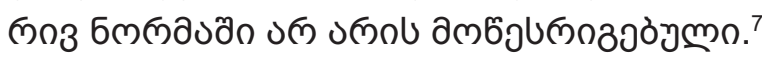

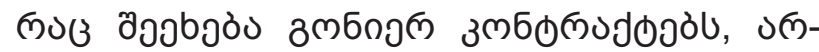

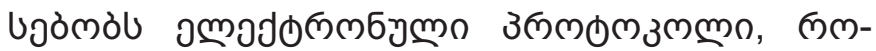

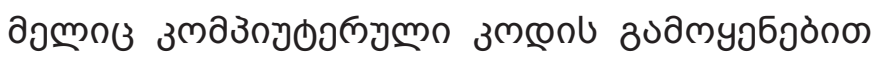

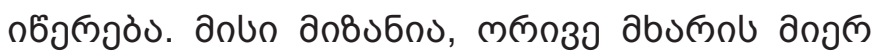

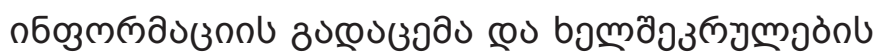

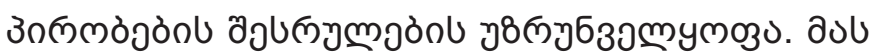

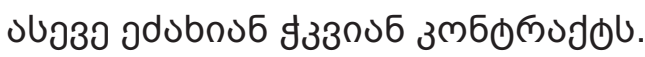

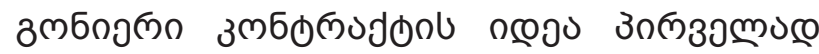

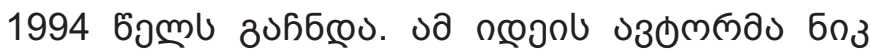

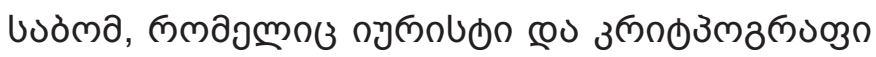

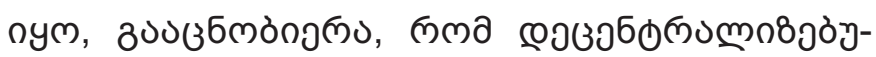

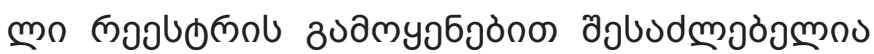

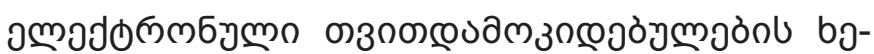

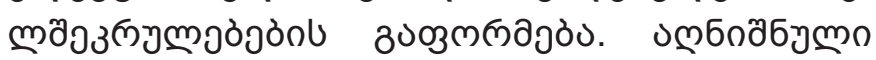

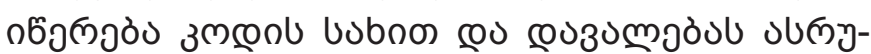

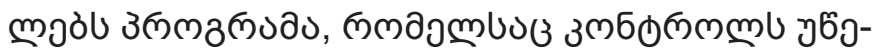

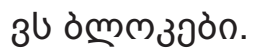

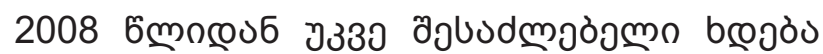

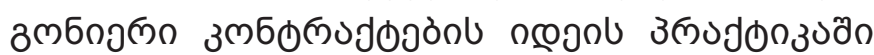

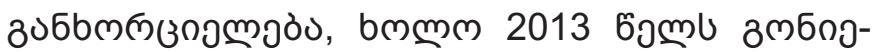

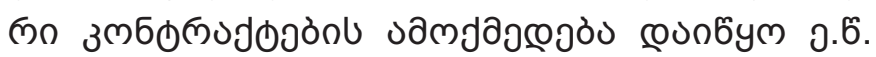

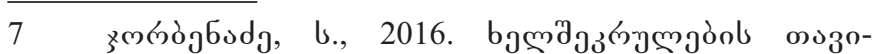

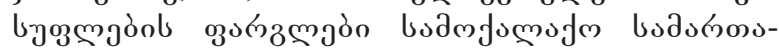
mə̀o. ó̀., 33 60-61. http://press.tsu.ge/ ob. Enders ,C., Wiederin, E., Rainer, P., Sodan, H., Der Sozialstaat in Deutschland und Europa, Peter M. Huber (Red.), De Gruyter Rechtswissenschaften Verlags-GmbH, Berlin, 2005, 365, Kittner M., Schuldrecht, Rechtliche Grundlagen - Wirschaftliche Zusammenhänge, 3. Auflage, Verlag Franz Vahlen, München, 2003, 161. 


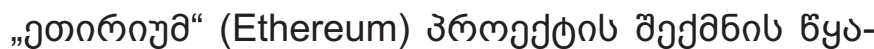

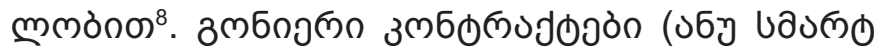

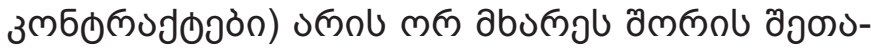

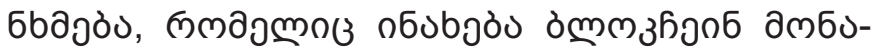

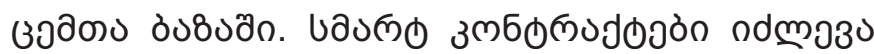

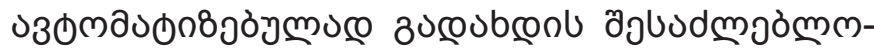

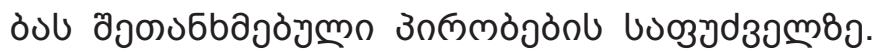

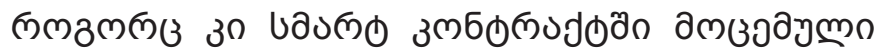

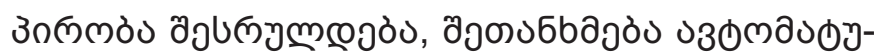

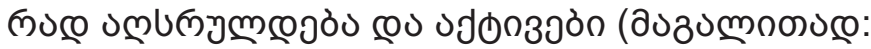

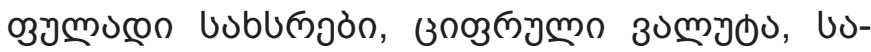

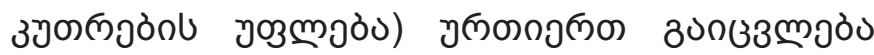

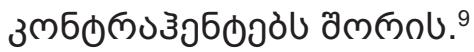

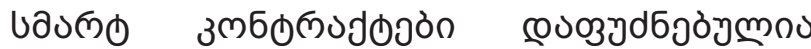

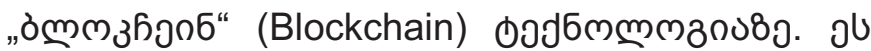

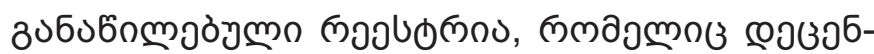

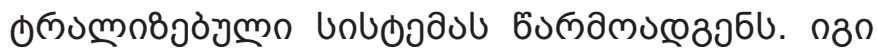

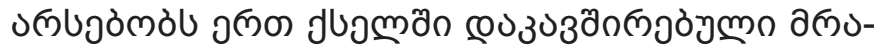

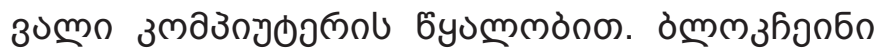

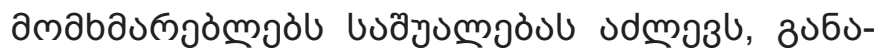

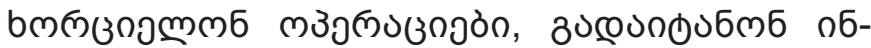

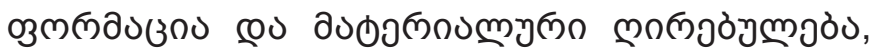

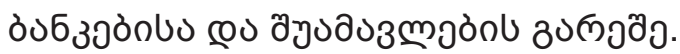

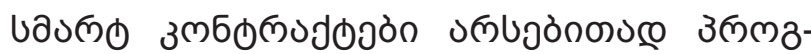

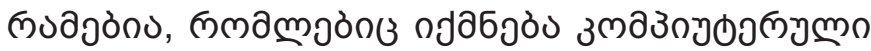

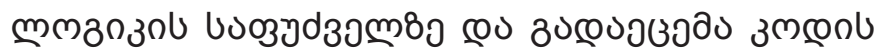

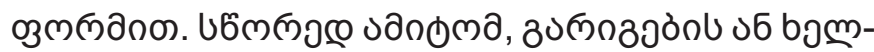

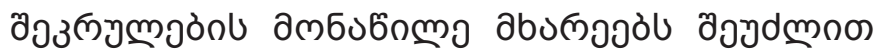

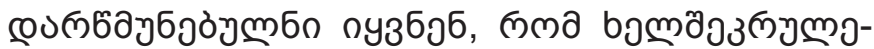

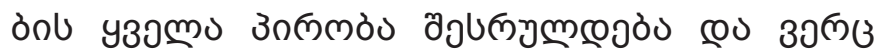

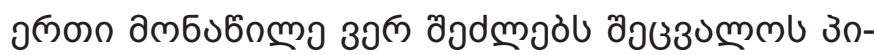

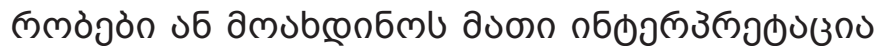

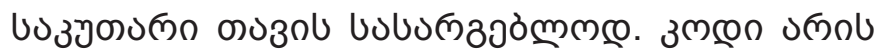

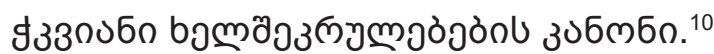

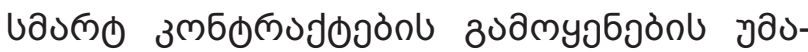

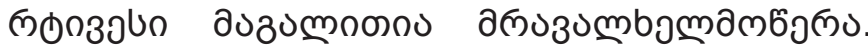

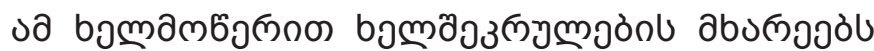

8

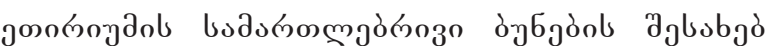

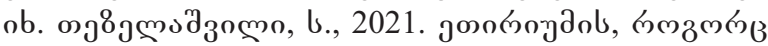

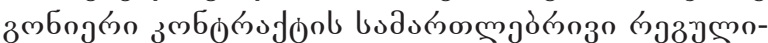

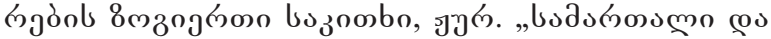

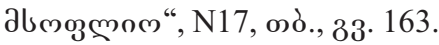

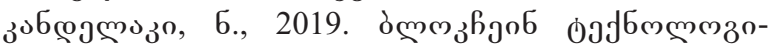

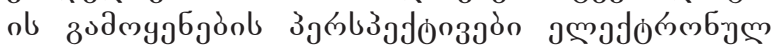

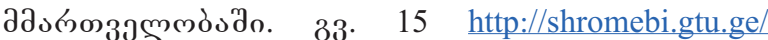
(ob. Iansiti, M., Lakhani, K. R., 2017. The truth about blockchain. Harvard Business Review. 118-127 pp;) (https://www.forbes.ru/tehnologii/343843-programmyblokcheyna-chtoprepyatstvuet-massovomuvnedreniyusmart-kontraktov).

10 https://www.blockchain-partners.com/ [4.12.2021]

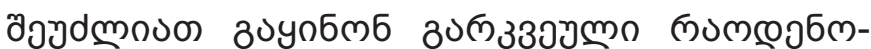

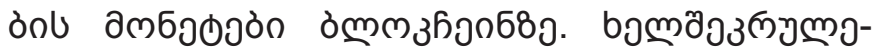

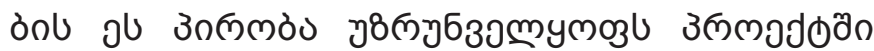

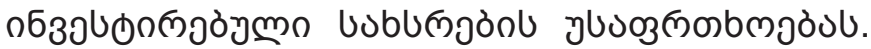

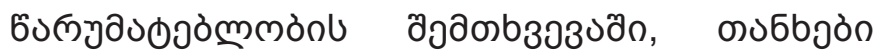

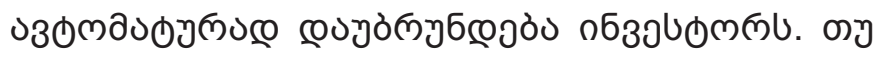

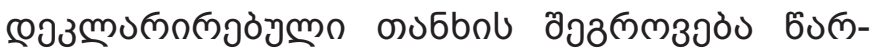

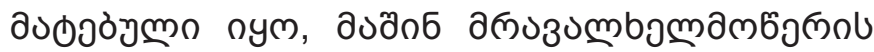

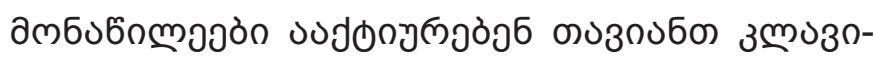

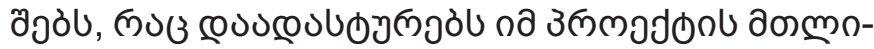

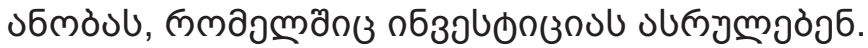

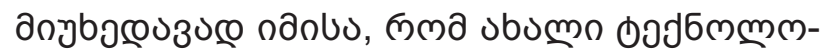

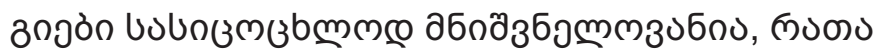

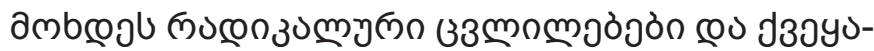

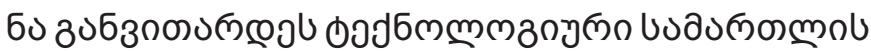

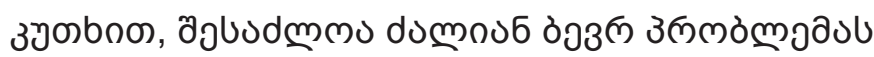

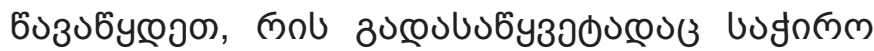

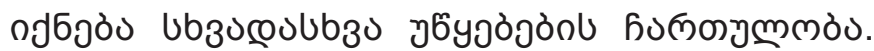

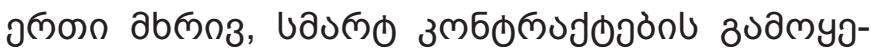

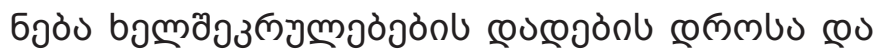

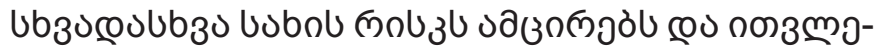

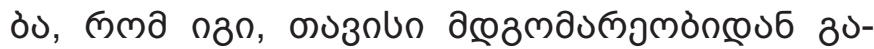

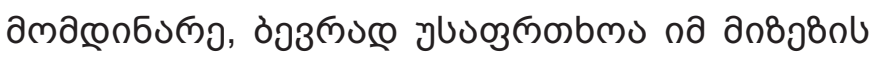

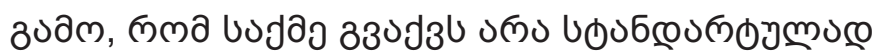

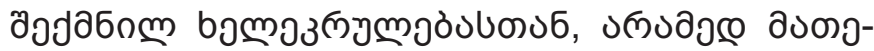

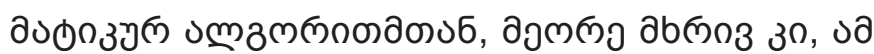

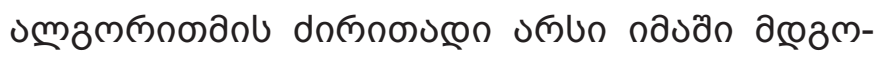

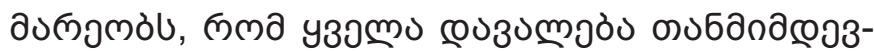

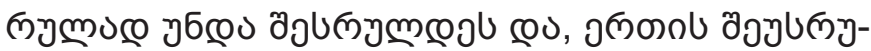

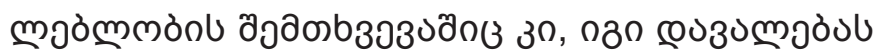

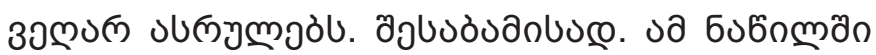

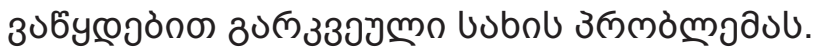

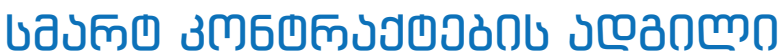

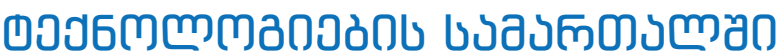

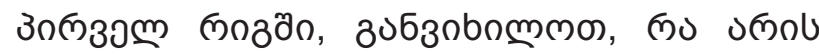

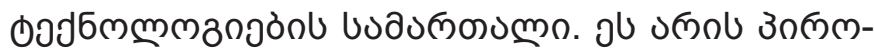

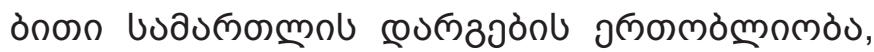

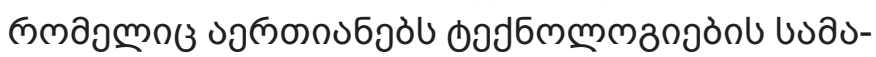

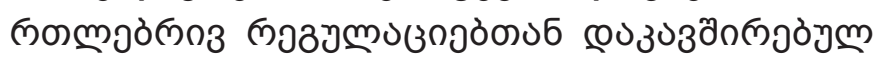

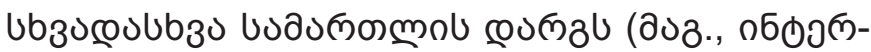

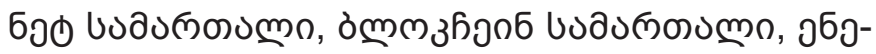

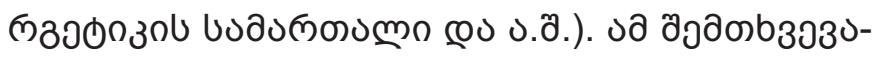

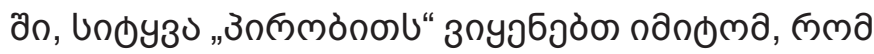

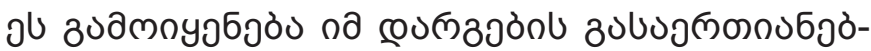

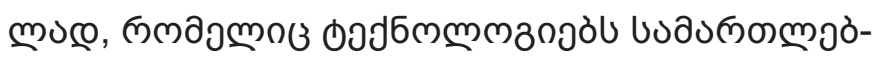

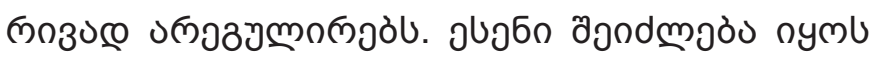




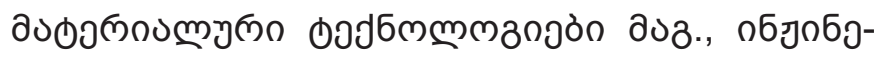

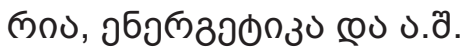

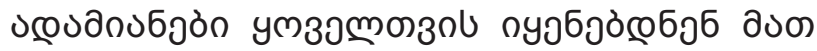

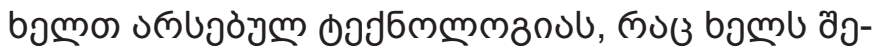

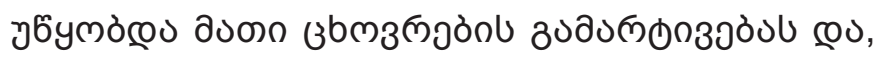

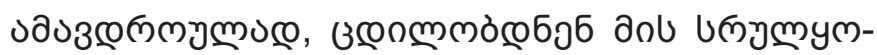

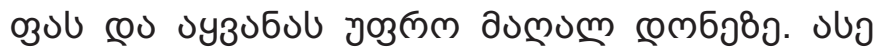

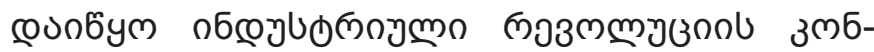
उ०Oु(300 11 .

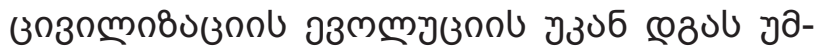

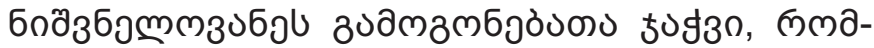

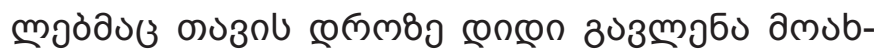

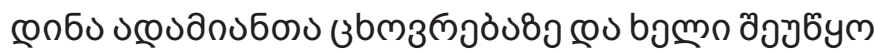

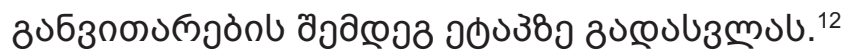

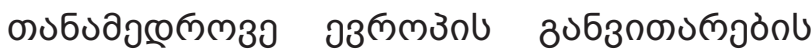

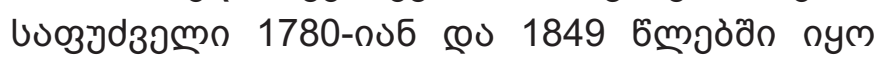

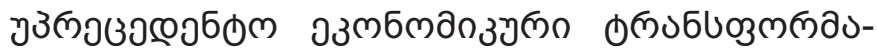

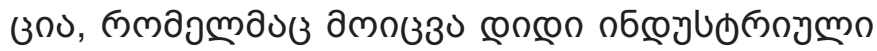

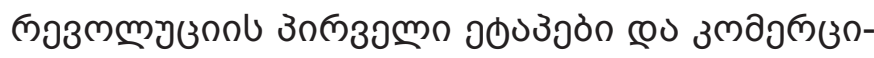

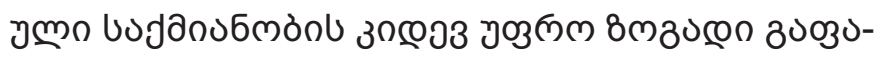

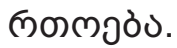

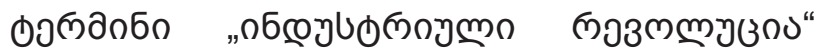

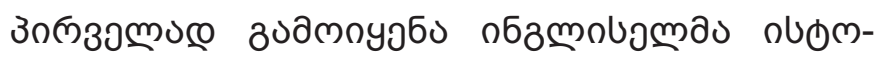

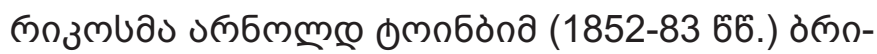

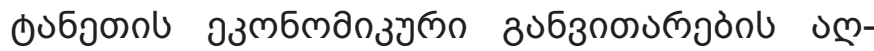

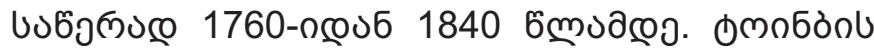

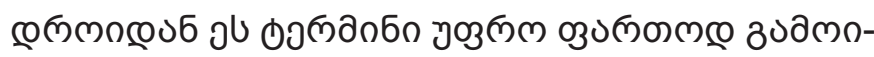

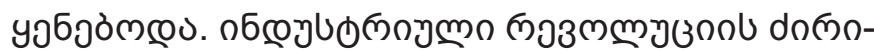

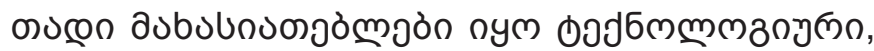

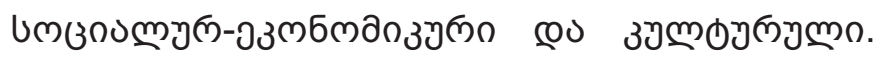

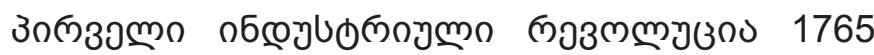

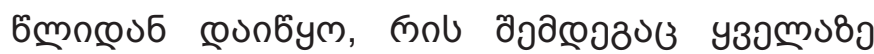

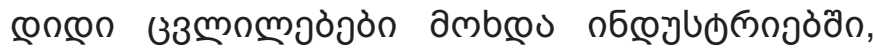

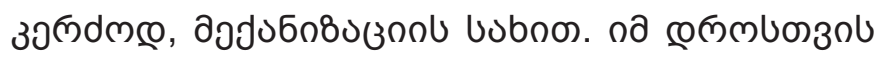

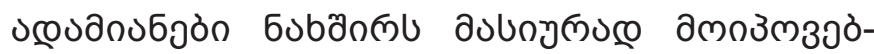

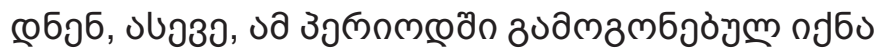

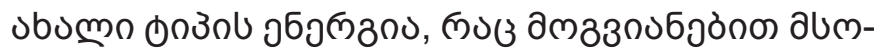

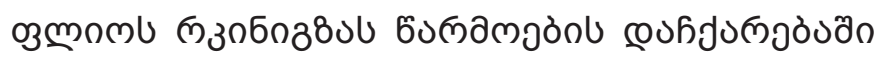

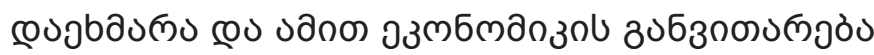

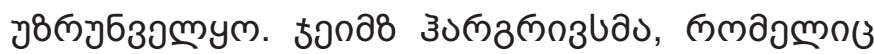

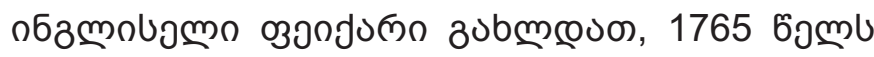

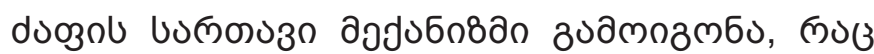

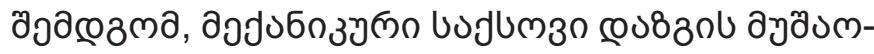

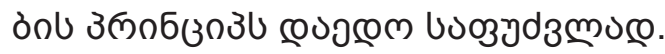

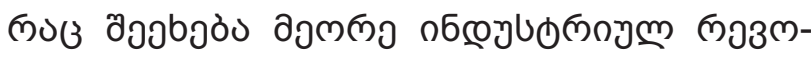

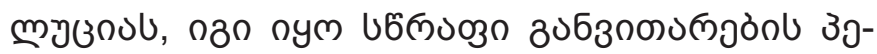

11 https://ied.eu/ [01.12.2020].

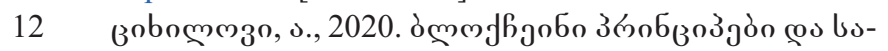

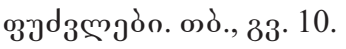

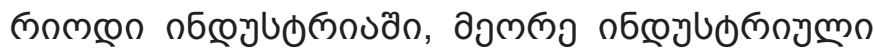

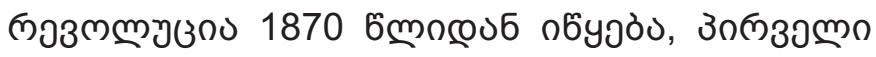

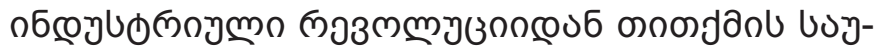

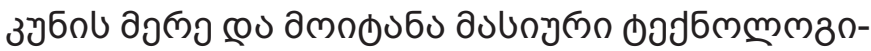

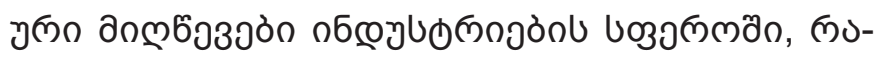

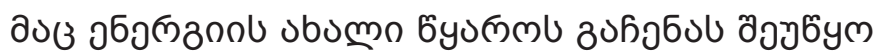

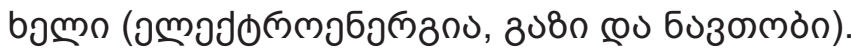

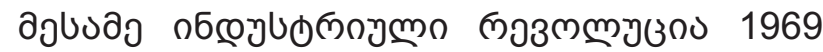

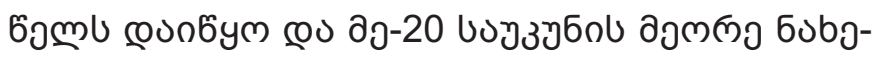

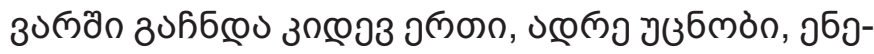

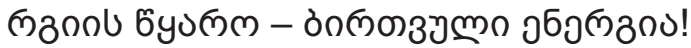

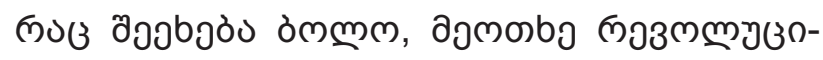

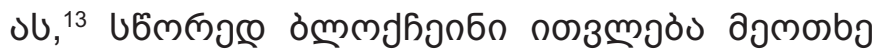

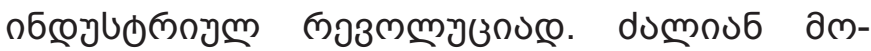

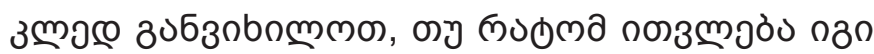

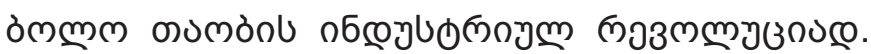

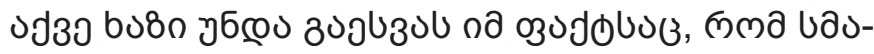

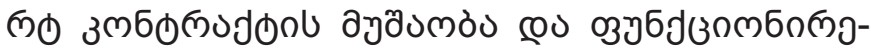

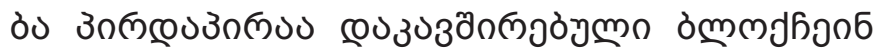

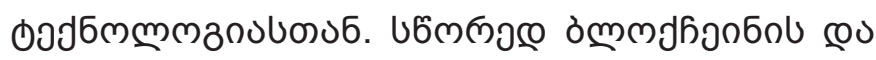

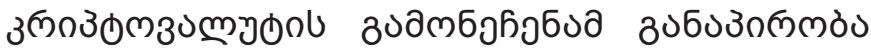

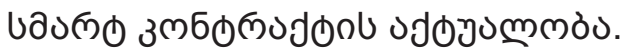

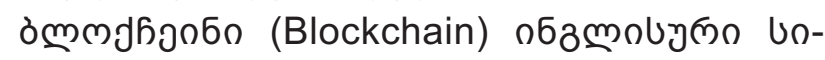

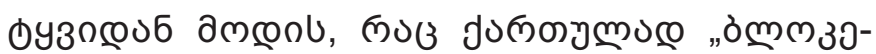

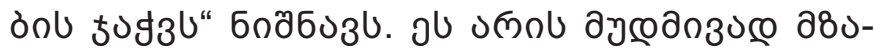

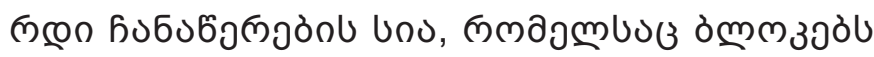

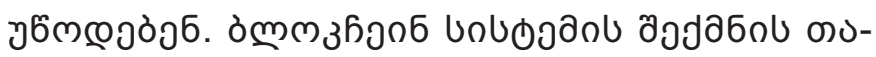

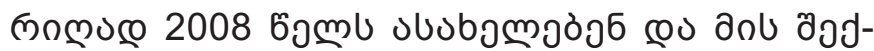

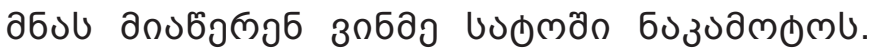

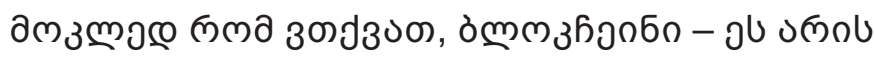

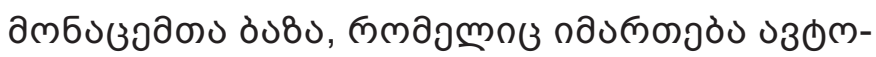

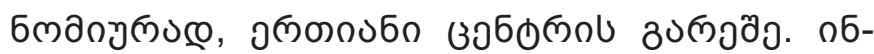

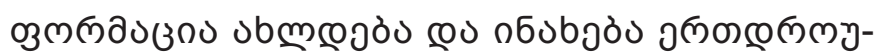

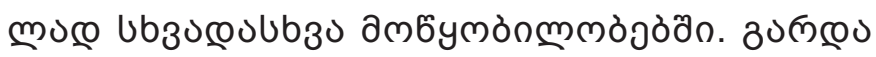

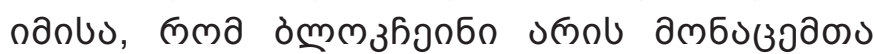

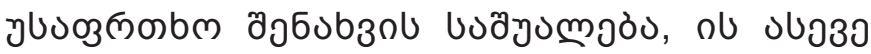

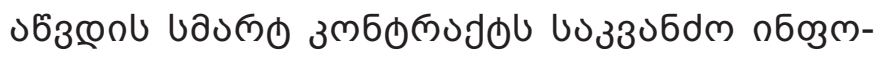

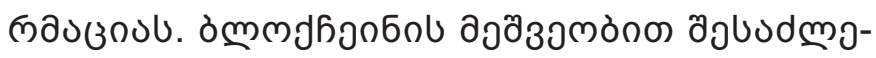

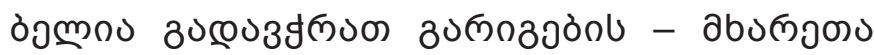

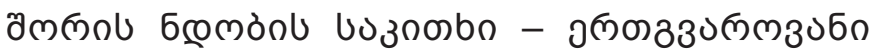

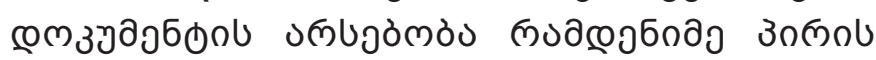

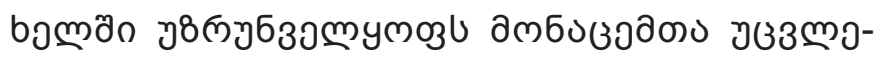

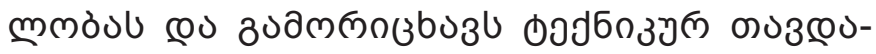
ubdjöb. ${ }^{14}$

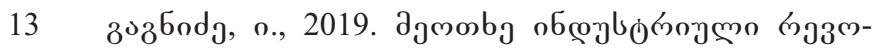

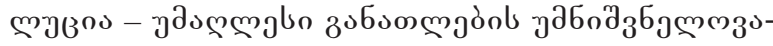

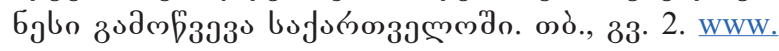
researchgate.net

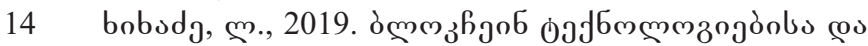

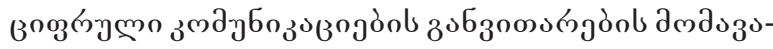




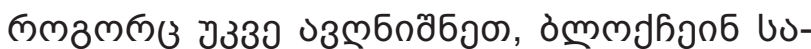

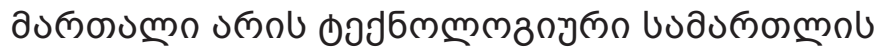

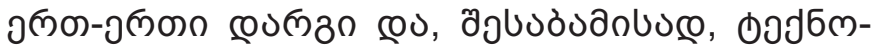

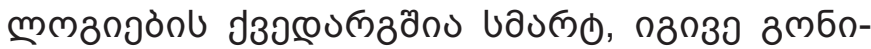

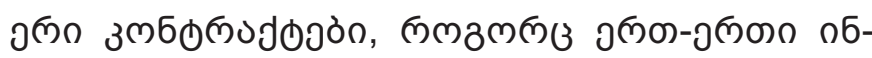

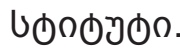

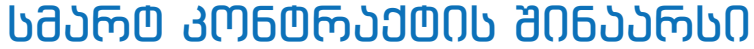

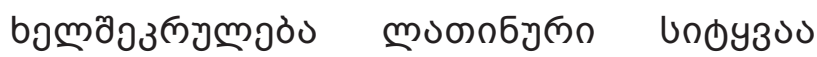
(moo. Contractus, Convention, Pactum; обо.

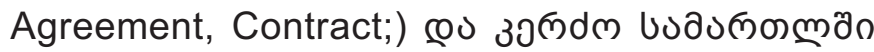

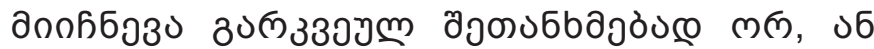

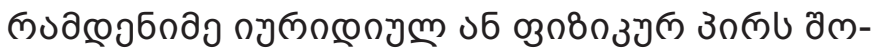

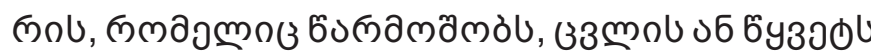

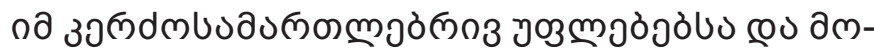

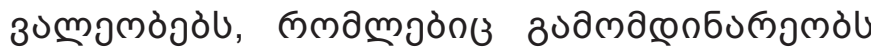

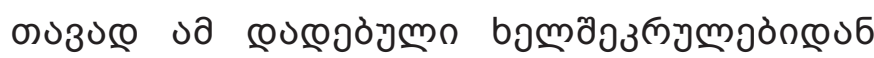

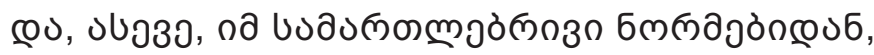

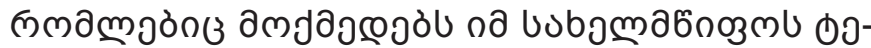

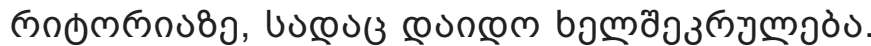

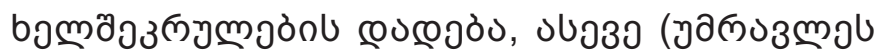

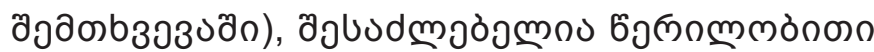

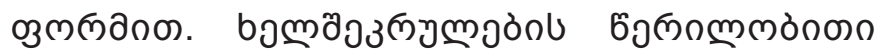

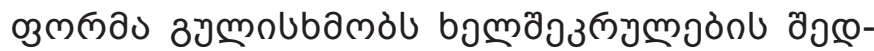

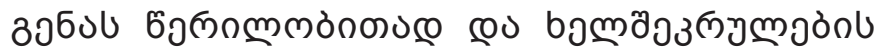

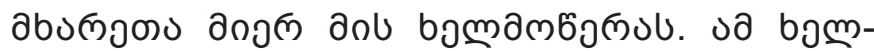

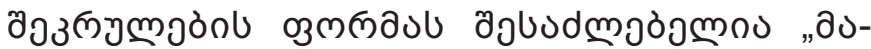

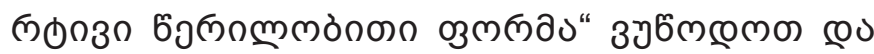

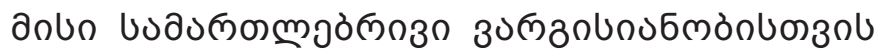

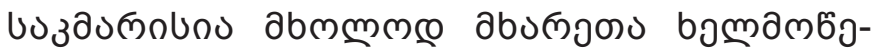

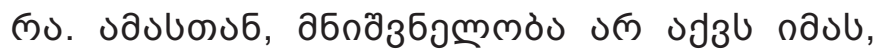

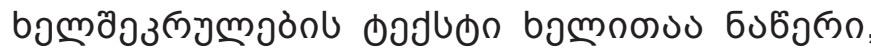

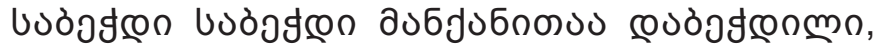

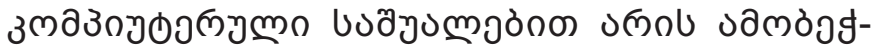

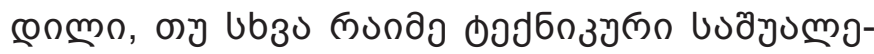

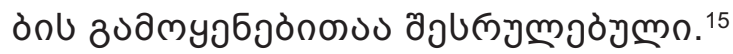

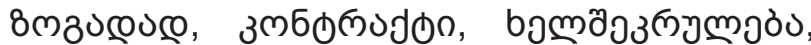

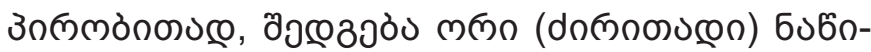

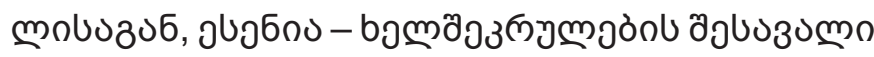

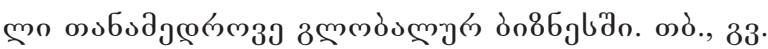
485. https://dspace.tsu.ge/ (ob. "8 ways blockchain can be an environmental game-changer", Celine Herweijer, Jahda Swanborough, Lead, Environment Initiatives, World Economic Forum www.weforum.org).

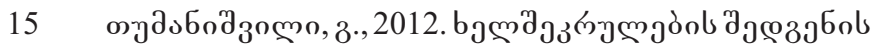

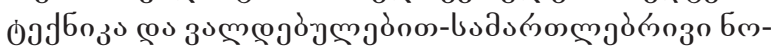

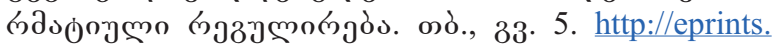
iliauni.edu.ge/

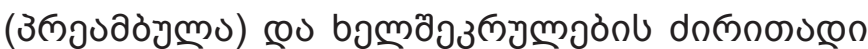

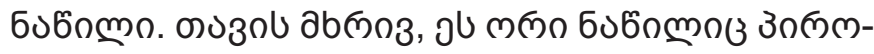

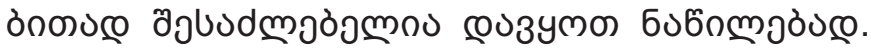

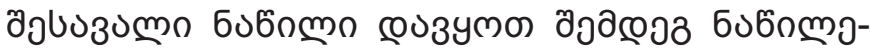

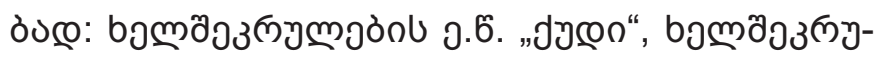

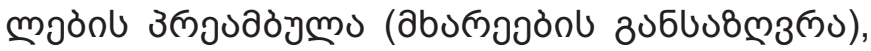

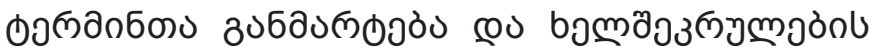

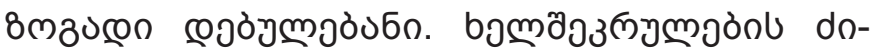

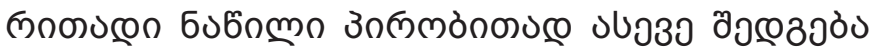

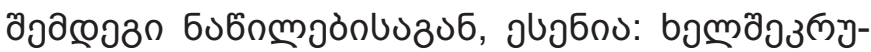

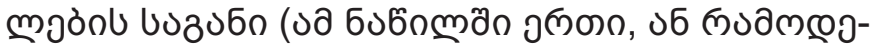

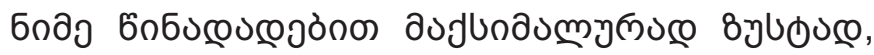

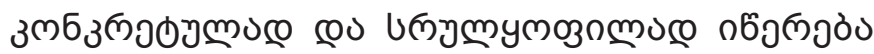

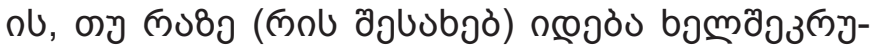

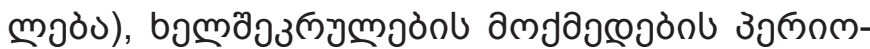

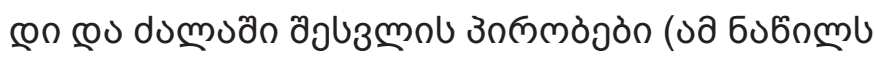

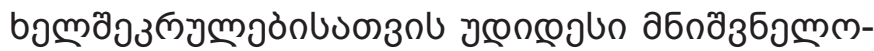

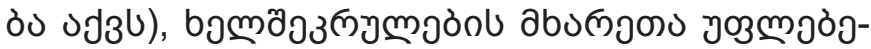

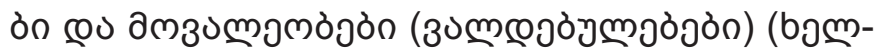

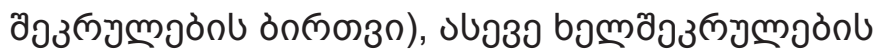

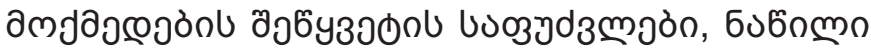

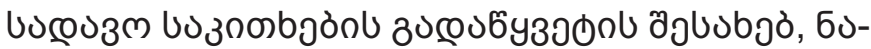

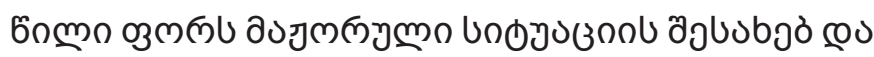

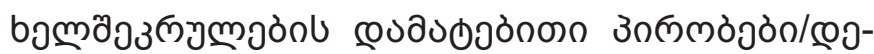

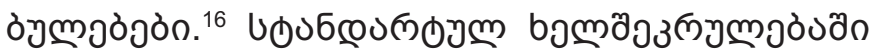

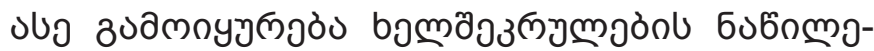

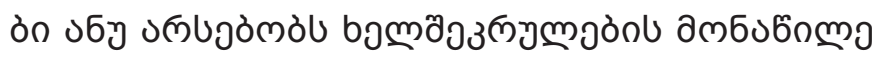

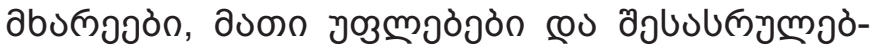

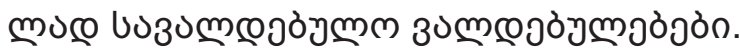

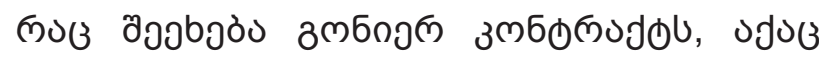

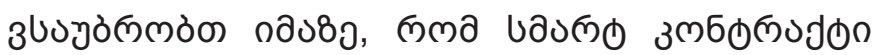

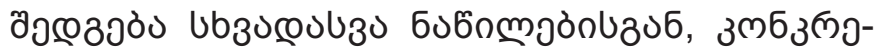

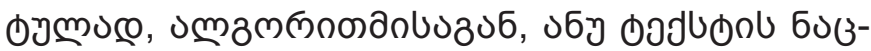

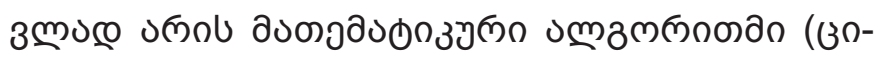

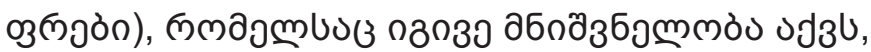

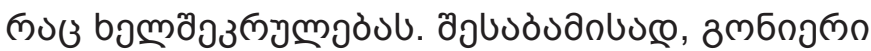

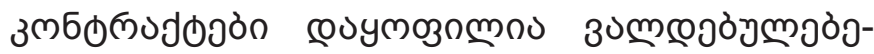

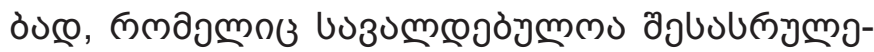

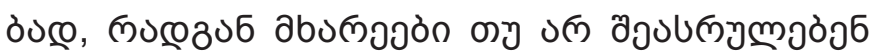

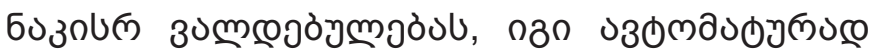

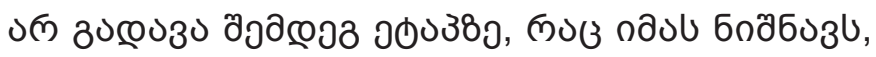

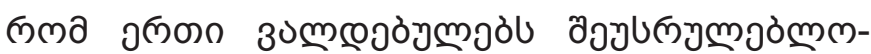

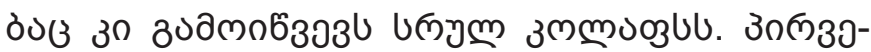

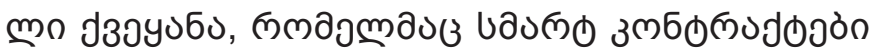

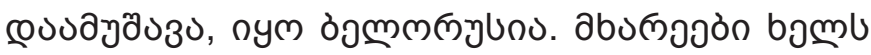

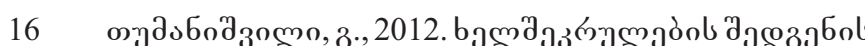

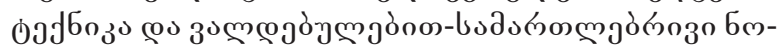

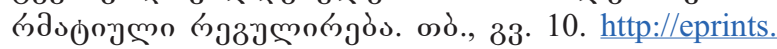
iliauni.edu.ge/ 


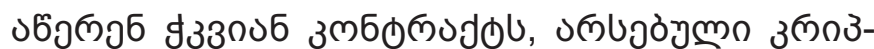

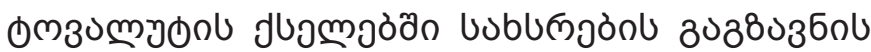

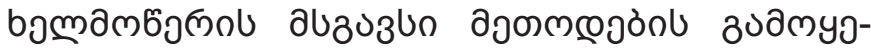

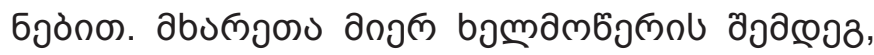

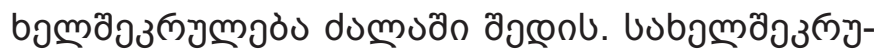

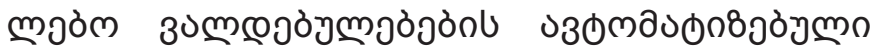

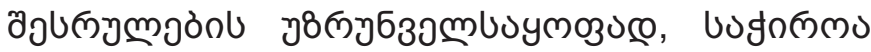
১ભ̆

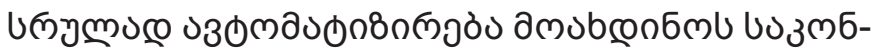

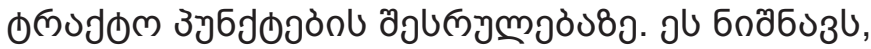

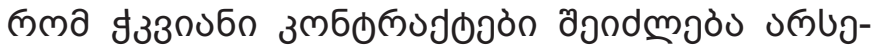

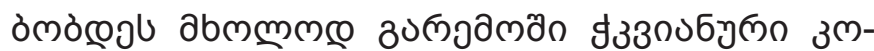

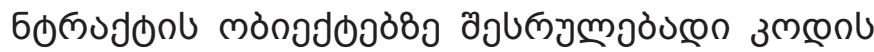

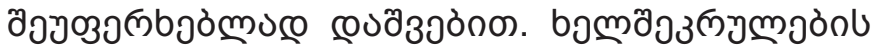

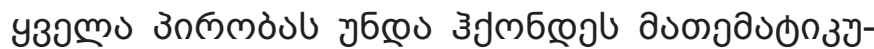

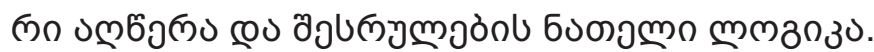

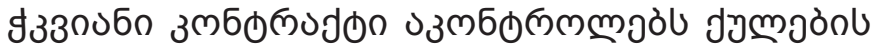

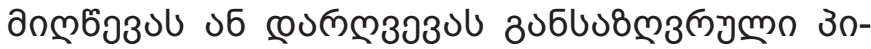

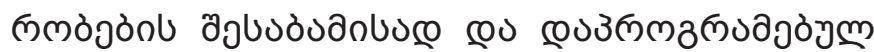

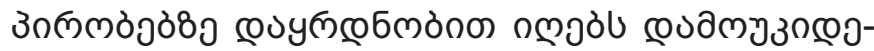

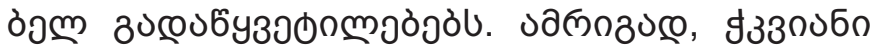

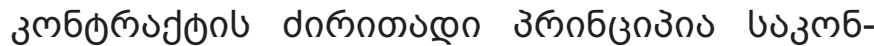

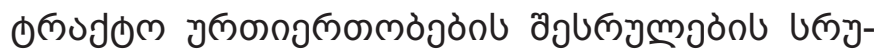

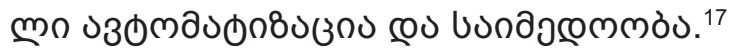

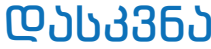

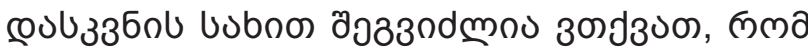

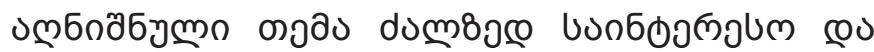

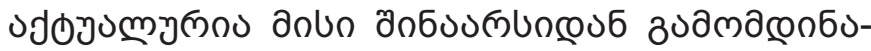

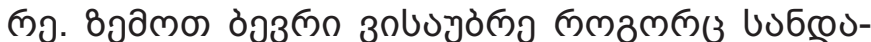

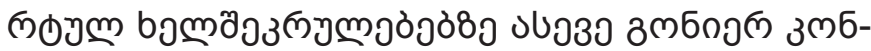

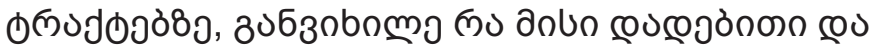

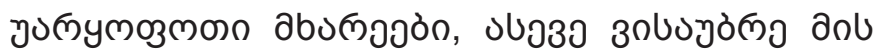

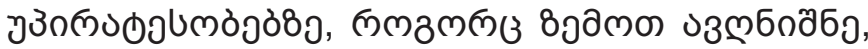

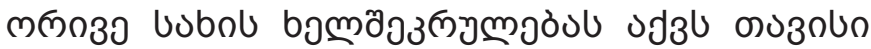

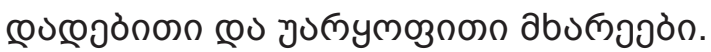

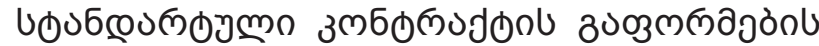

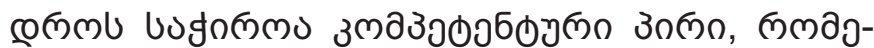

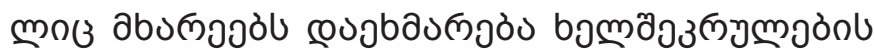

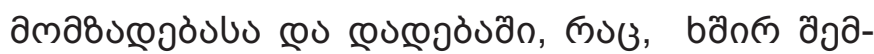

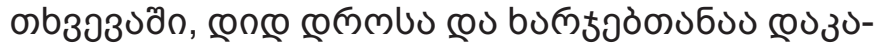

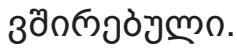

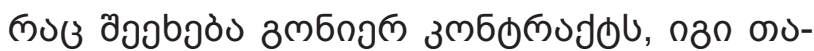

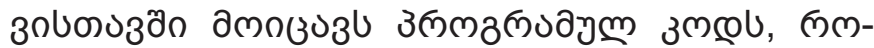

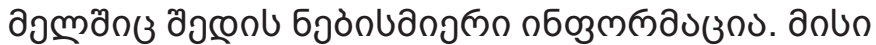

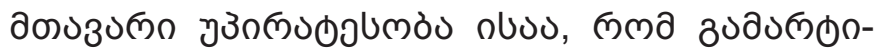

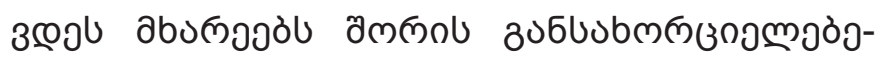

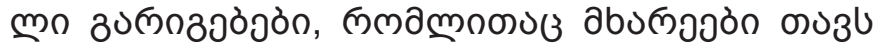

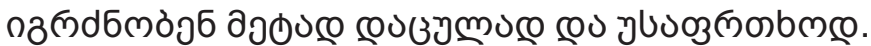

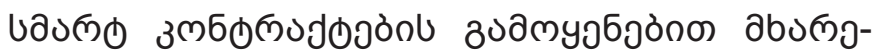

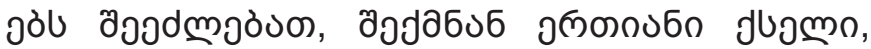

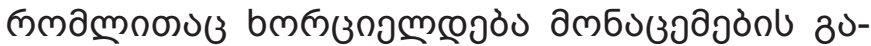

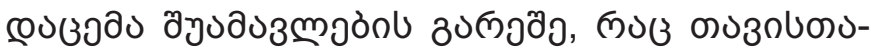

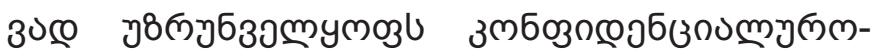

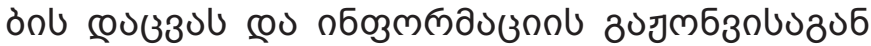

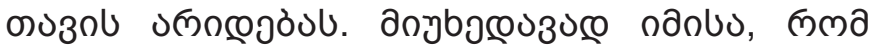

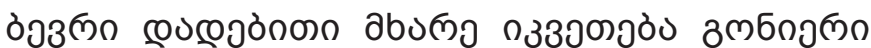

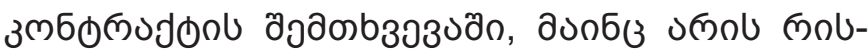

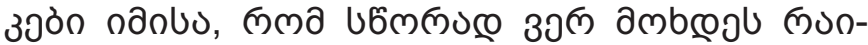

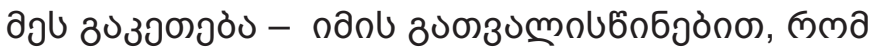

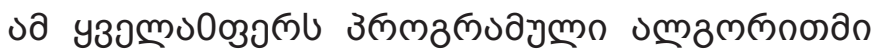

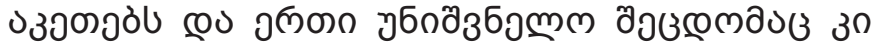

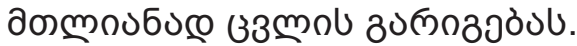

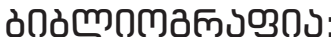

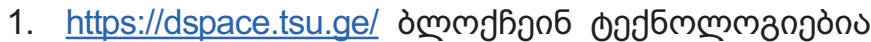

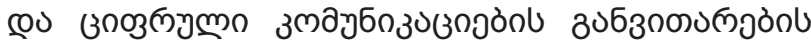

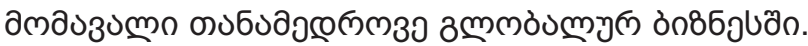
bubodo, $m$.

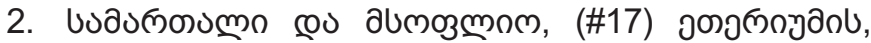

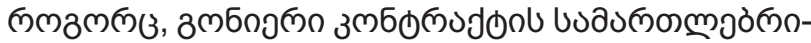

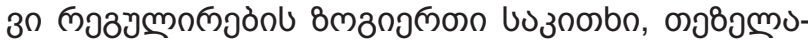
əзомо, U.

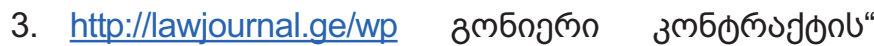

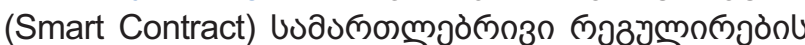

\section{BIBLIOGRAPHY:}

1. https://dspace.tsu.ge/ Blockchain technologies and the future of development the digital communications in modern global business. Khakhadze, L.

2. Law and World, (\# 17) Some Issues of Legal Regulation of Etherium as a Wise Contract, Tezelashvili, S.

3. http://lawjournal.ge/wp Some Conceptual Issues of the Smart Contract' Legal Regulation. Gabisonia, $Z$.

4. http://lawlibrary.info/ge Commentary on the Civil 


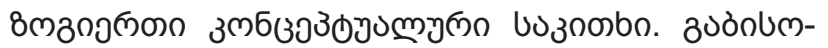
ธกง, 8.

4. http:/lawlibrary.info/ge b১amjumodm зmূоodunb

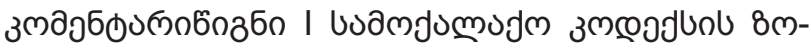

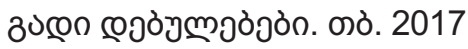

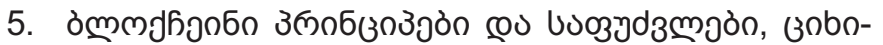
mल3n, ১. कठ̀ 2020

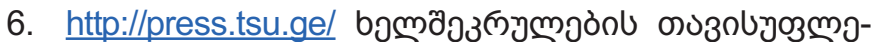

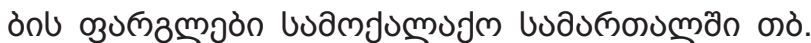

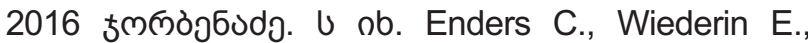
Rainer P., Sodan H., Der Sozialstaat in Deutschland und Europa, Peter M. Huber (Red.), De Gruyter Rechtswissenschaften Verlags-GmbH, Berlin, 2005, 365, Kittner M., Schuldrecht, Rechtliche Grundlagen - Wirschaftliche Zusammenhänge, 3. Auflage, Verlag Franz Vahlen, München, 2003

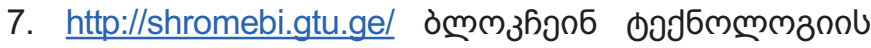

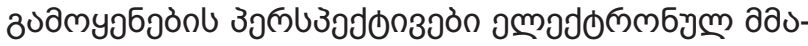

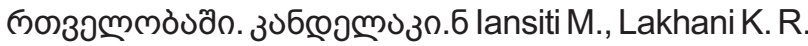
The truth about blockchain. Harvard Business Review. 2017, URL: https://www.forbes.ru/tehnologii/343843programmy-blokcheyna-chtoprepyatstvuetmassovomuvnedreniyu-smart-kontraktov

8. https://www.blockchain-partners.com

9. https://ied.eu

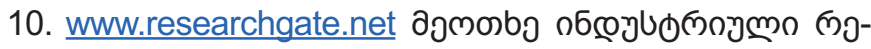

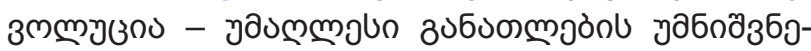

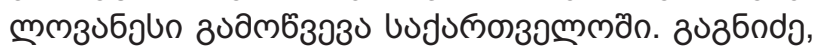
0 .

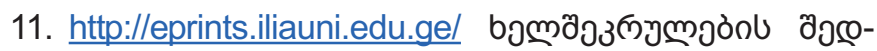

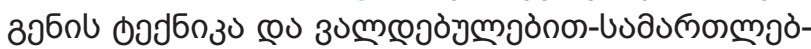

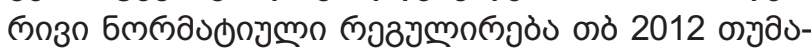

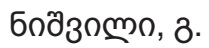

12. https://milloin-money.webnode.com
Code. Book I General provisions of the Civil Code. Tbilisi 2017

5. Blockchain Principles and Fundamentals Tsikhilovi, A. Tbilisi 2020

6. http://press.tsu.ge/ Scope of freedom of contract in civil law Tbilisi 2016, Jorbendze. S Enders C., Wiederin E., Rainer P., Sodan H., Der Sozialstaat in Deutschland und Europa, Peter M. Huber (Red.), De Gruyter Rechtswissenschaften Verlags-GmbH, Berlin, 2005, 365, Kittner M., Schuldrecht, Rechtliche Grundlagen - Wirschaftliche Zusammenhänge, 3. Auflage, Verlag Franz Vahlen, München, 2003

7. http://shromebi.gtu.ge/ Prospects of using the blockchain technology in e-governmenSEVERAL ASPECTS IN THE LEGAL REGULATION OF ETHEREUM AS THE „SMART CONTRACTSce. Kandelaki. N. page 15 (1. lansiti M., Lakhani K. R. The truth about blockchain. Harvard Business Review. 2017, URL: https://www.forbes.ru/tehnologii/343843-programmy-blokcheyna-chtoprepyatstvuet-massovomuvnedreniyu-smart-kontraktov

8. https://www.blockchain-partners.com/ Several aspects in the legal regulation of eterium as the smart contract page

9. https://ied.eu/

10. www.researchgate.net The $4^{\text {th }}$ Industrial Revolution - The most important challenge of higher education in Georgia. Gagnidze, I.

11. http://eprints.iliauni.edu.ge/ Contract creating technique and obligation-legal normative regulation Tbilisi 2012. Tumanishvili, G.

12. https://milloin-money.webnode.com

\section{NOTES:}

1. https://dspace.tsu.ge/ Blockchain technologies and the future of development the digital communications in modern global business. Khakhadze, Tbilisi, 2019, p. 585

2. Law and World, (\# 17) Some Issues of Legal Regulation of Etherium as a Wise Contract, Tezelashvili, S. Tbilisi, 2021, p. 168

3. http://lawjournal.ge/wp Some Conceptual Issues of the Smart Contract' Legal Regulation. Gabisonia Z. Tbilisi, 2020, p. 45

4. http://lawlibrary.info/ge Commentary on the Civil Code. Book I General provisions of the Civil Code. Tbilisi, 2017, p. 287

5. http://lawlibrary.info/ge Commentary on the Civil Code. Book III General Part of The law of obligations. Tbilisi, 2019, pp. 108,109

6. Blockchain Principles and Fundamentals Tsikhilovi, A. Tbilisi, 2020, p. 10

7. http://press.tsu.ge/ Scope of freedom of contract in civil law Tbilisi 2016, Jorbendze. S page 42-43 Enders C., Wiederin E., Rainer P., Sodan H., Der Sozialstaat in Deutschland und Europa, Peter M. Huber (Red.), De Gruyter Rechtswissenschaften Verlags-GmbH, Berlin, 2005, 365, Kittner M., Schuldrecht, Rechtliche Grundlagen - Wirschaftliche Zusammenhänge, 3. Auflage, Verlag Franz Vahlen, München, 2003, 161 
8. http://shromebi.gtu.ge/ Prospects of using the blockchain technology in e-governmenSEVERAL ASPECTS IN THE LEGAL REGULATION OF ETHEREUM AS THE „SMART CONTRACTSce. Kandelaki. N. page 15 (1. lansiti M.,Lakhani K. R. The truth about blockchain. Harvard Business Review. 2017, 118-127 pp; 2. URL: https://www.forbes.ru/tehnologii/343843-programmy-blokcheyna-chtoprepyatstvuet-massovomuvnedreniyu-smart-kontraktov

9. https://lawandworld.ge/ Several aspects in the legal regulation of eterium as the smart contract, p. 163

10. https://www.blockchain-partners.com/

11. https://ied.eu/ Date of last access 01.12.2020

12. Blockchain Principles and Fundamentals Tsikhilovi, A. Tbilisi, 2020, p. 10

13. www.researchgate.net The $4^{\text {th }}$ Industrial Revolution - The most important challenge of higher education in Georgia. Gagnidze, I p. 2

14. https://dspace.tsu.ge/ Blockchain technologies and the future of development the digital communications in modern global business. Khakhadze, L., Tbilisi, 2020, p. 485, 8 ways blockchain can be an environmental game-changer", Celine Herweijer,Jahda Swanborough, Lead, Environment Initiatives, World Economic Forum. www.weforum.org)

15. http://eprints.iliauni.edu.ge/ Contract creating technique and obligation-legal normative regulation. Tbilisi, 2012. Tumanishvili p. 5

16. http://eprints.iliauni.edu.ge/ Contract creating technique and obligation-legal normative regulation. Tbilisi, 2012. Tumanishvili p. 10

17. https://milloin-money.webnode.com/ 